\title{
Marine fungi of Div Island (India): Basidiomycota and Mitosporic fungi V.M. Kambale ${ }^{1}$ and *S.A. Firdosi
}

${ }^{1}$ Dr. Annasaheb J. D. Bendale Mahila Mahaidyalaya, JALGAON (M.S.) INDIA

H. J. Thim Arts \& Science College, JALGAON, Dist. JALGAON (M.S.) INDIA ${ }^{*}$ Corresponding Author Email : shakeel.talk@gmail.com

Received : 25.08.2020; Accepted : 22.09.2020

\begin{tabular}{l} 
ABSTRACT \\
The present paper deals with two species of Basidiomycetes and seven species of Mitosporic fungi encountered on \\
intertidal woody debris from coast of Div Island (Arabian sea, India). Basidiomycetes include: Halocyphina villosa Kohlm. \& E. \\
Kohlm. and Nia vibrissa R.T. Moore \& Meyers. Mitosporic fungi include: Cirrenalia basiminuta Raghukumar \& Zainal, Clavatospora \\
bulbosa (Anastasiou) Nakagiri \& Tubaki, Hydea pygmea (Kohlm.) K.L. Pang \& E.B.G. Jones, Matsusporium tropicale (Kohlm.) \\
E.B.G.Jones \& K.L. Pang, Trichocladium achrasporum (Meyers and R.T.Moore) Dixon, T. alopallonellum (R.T. Moore \& Meyers) \\
Kohlm. \& V.-Kohlm. and T. lignicola I. Schmidt. The data provide information on the distribution of these fungi from India, apart \\
from their description and illustrations. \\
\hline Figures : 09 References : 10 \\
KEY WORDS : Basidiomycetes, Intertidal wood, Marine, Mitosporic fungi.
\end{tabular}

\section{Introduction}

Coastal wetlands are considered as the most productive natural marine ecosystems. Biodiversity of marine fungi of the world is well documented. Diversity of marine fungi of India is also documented. Filamentous Basidiomycota as a group is rare in the marine habitats, with only 21 species (in 17 genera) are known. Marine Mitosporic fungi (Asexual forms) occur on a wide variety of substrates in oceans and estuaries. Hosts or substrates include algae, submerged parts of mangroves and salt marsh halophytes, intertidal woody debris, and animal chitin.

\section{Materials and Methods}

Studies on marine fungi from Div Island were made. All these encountered fungi have been recorded for the first time from Div Island coast, described and illustrated in this paper.

During marine mycological survey of the coast of Div Island (Arabian sea, India), two species of Basidiomycetes and seven species of Mitodporic fungi were collected on samples of intertidal woody debris.

The samples of intertidal woody debris were collected at low tide, in polythene bags from the coast of
Div Island (Arabian Sea, India) and brought to the laboratory. Samples were examined for fungal growth. Then they were incubated at room temperature in plastic boxes for few months and the fungi growing on them were identified. The slides were made semi-permanent by using double cover glass method ${ }^{10}$. Identification of encountered fungi and records from Div Island were confirmed with the help of literature ${ }^{2,4,6}$.

\section{Systematic Account}

1) Halocyphina villosa Kohlm. \& E. Kohlm, 1965. Nova Hedwigia, 9: 100.

Basidiomes: cyphelloid, stalked, gregarious, turbinate, funnel-shaped, superficial, whitish, soft, thin-walled, tomentous. Basidiospores: 8-9 x 8-9 $\mu \mathrm{m}$, subglobose, one-celled, smooth, hyaline, non-amyloid, accumulating at maturity in the opening of the basidiome.

Distribution in India:- West Coast: Gujarat, Maharashtra, Goa, Karnataka, Puducherry (Mahe), Kerala, Lakshadweep Islands; East Coast: Tamil Nadu, Puducherry (Karaikkal), Andhra Pradesh, Odisha, Andaman and Nicobar Islands (see 2,3,\& 4.)

2) Nia vibrissa R.T. Moore \& Meyers, 1959. Mycol., 51 : 874. 
Basidiomes: gregarious, subglobose, superficial, yellowish, pinkish, and finally orange-coloured, soft, thinwalled, villose or smooth. Basidiospores: 10-15 x 6-10 $\mu \mathrm{m}$, ovoid or ellipsoidal, one-celled, hyaline, with several slender appendages. Appendages: at the apex provided with a single, slender, flexible, attenuate, hyaline appendage, $25-45 \times 1 \mu \mathrm{m}$, terminally slightly inflated; four (rarely 3 or 5) similar, sub-terminal, radiating appendages around the base, $20-30 \mu \mathrm{m}$ long, with a short cylindrical projection at the basal region.

Distribution in India:- West Coast: Gujarat, Maharashtra, Goa, Karnataka, Kerala; East Coast:Tamil Nadu, Andhra Pradesh, West Bengal, Odisha (see, 2,3,\& 4).

3) Cirrenalia basiminuta Raghukumar \& Zainal, 1988. Mycotaxon, 31: 163.

Conidia: 3-5-septate, constricted at the septa. Conidial cells increasing in size from base to apex, pale brown. Apical cell 9-15 x 9-13 $\mu \mathrm{m}$, subglobose, basal cell cylindrical and tapering, 5-171 x 3-7 $\mu \mathrm{m}$. Pigmentation of the cells increasing from base to apex, the apical cell light brown with a reddish tinge.

Distribution in India:- West Coast: Gujarat, Maharashtra, Goa, Karnataka, Kerala, Lakshadweep Islands, East Coast:Tamil Nadu, Andhra Pradesh, Odisha (see, 2,3\& 4).

4) Clavatospora bulbosa (Anastasiou) Nakagiri and Tubaki, 1985. Bot.Mar., 28: 489.

Conidia: tetraradiate, septate, slightly constricted at the septa, hyaline to light brown, developing by transformation of the inflated apex of the conidiophore; basal arm one-septate; proximal cell 10-20 x 5-11 $\mu \mathrm{m}$, ellipsoidal or ovoid, truncate at the base, light brown; distal cell 7-13 x 7-12 $\mu \mathrm{m}$, cylindrical or shortly three branched, fuscous; three divergent arms arising simultaneously from the inflated distal cell of basal arm, 20-70 x 4-6 $\mu \mathrm{m}$, cylindrical, one- to seven-septate, light brown. One armed conidia: Conidia consisted of only single arm also observed, these are grey brown, 5-10 celled, 55-60 x 7-9 $\mu \mathrm{m}$, constricted at the septa, basal and apical cells are with lighter colour.

Distribution in India:- West Coast: Gujarat, Maharashtra, Goa, Karnataka, Puducherry (Mahe), Lakshadweep Island; East Coast: Tamil Nadu, Puducherry, Andhra Pradesh, West Bengal. Andaman \& Nicobar Islands (see, 2,3,\& 4).

5) Hydea pygmea (Kohlm.) K.L. Pang \& E.B.G. Jones, 2010. In: Abdel-Wahab et al., Mycol. Progress, 9: 549.

Conidia: acrogenous, solitary, igantean, contorted $1 / 2$ or 1 time contorted, 3-4-septate, not or slightly constricted at the septa, hooked appearance, black or fuscous, fulgent (upper three cells dark, lower two or three cells light-coloured); cells increasing in diameter from base to apex, distinctly dissimilar; spirals $25-31$ x 28-34 $\mu \mathrm{m}$; terminal cell $16-23 \mu \mathrm{m}$ in diam, subglobose to reniform, basely flattened; basal cells $4-5 \mu \mathrm{m}$ in diam; central cells irregularly conical or almost wedge-shaped.

Distribution in India:- West Coast: Gujarat, Maharashtra, Goa, Karnataka, Puducherry (Mahe), Kerala, Lakshadweep Islands; East Coast: Tamil Nadu, Puducherry, Andhra Pradesh, West Bengal, Andaman \& Nicobar Islands (see,2,3, \& 4).

6) Matsusporium tropicale (Kohlm.) E.B.G. Jones \& K.L. Pang, 2010. In: Abdel-Wahab et al., Mycol. Progress, 9: 550.

Conidia: acrogenous, solitary, regularly or irregularly igantean, mostly 1 to $1 \frac{1}{2}$ times contorted, rarely semicontorted, 5-10-septate, not or slightly constricted at the septa, reddish brown; cells increasing in diameter from base to apex, distinctly dissimilar; spirals 23-30 $\mu \mathrm{m}$ in diameter; terminal cell 8-14 $\mu \mathrm{m}$ high, 10-13 $\mu \mathrm{m}$ diam, subglobose to ellipsoidal, basally flattened; basal cells 8-17 $\mu \mathrm{m}$ high, 3-5 $\mu \mathrm{m}$ in diam, cylindrical; central cells subglobose, obtusely conical or dolliform.

Distribution in India:- West Coast: Maharashtra, Goa, Karnataka, Kerala, Lakshadweep Islands; East Coast: Tamil nadu, Puducherry (Karaikkal), Andhra Pradesh, Odissa, West Bengal, Andaman \& Nicobar Islands (see, 2,3,\&4).

7) Trichocladium achrasporum (Meyers and R.T. Moore) Dixon, 1971. In: Shearer \& Crane, Mycologia, 63: 344.

Conidia: $20-32 \times 10-23 \mu \mathrm{m}$, clavate, ovoid or obpyriform, 2-4-septate, less constricted at the septa, straight or slightly curved, increases in diameter from base to apex, formed singly on the conidiophores; apical cells subglobose, dark brown; basal cells conical or subcylindrical, subhyaline to light brown or fuscous.

Distribution in India:- West Coast: Gujarat, Maharashtra, Karnataka, Puducherry (Mahe), Kerala, Lakshadweep Islands; East Coast: Tamil Nadu. Andhra Pradesh, Odisha, West Bengal, Andaman \& Nicobar Islands (see, 2,3, \& 4).

8) Trichocladium alopallonellum (R.T. Moore \& Meyers) Kohlm. \& Volkm.-Kohlm., 1995. Mycotaxon, 53: 352.

Conidia: 10-20 x 8-16 $\mu \mathrm{m}$, obpyriform, ovoidal or subglobose, one- to two-celled, fuscous; when two-celled, apical cell larger ( 9-15 x 7-12 $\mu \mathrm{m}$ ), ovoid, fuscous, basal cell smaller, obconical to cylindrical, light brown, conidiogenous cell usually remaining connected with the 
ㅎํㄴ
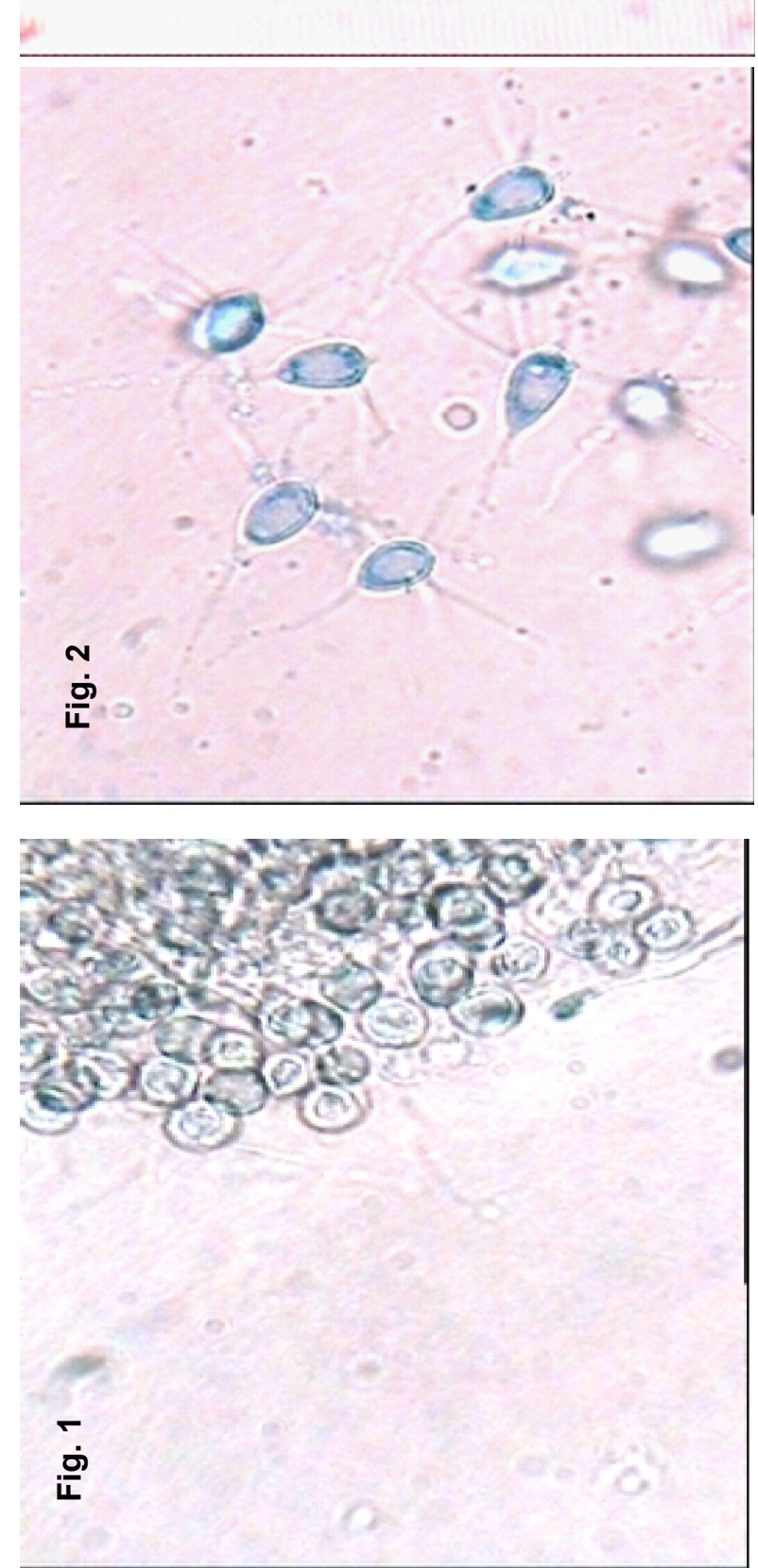

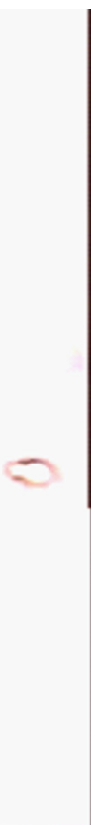

n)

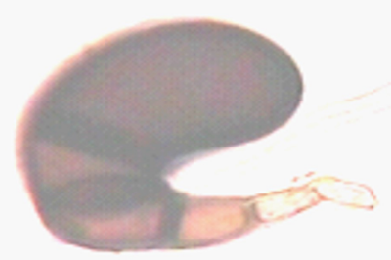

흐

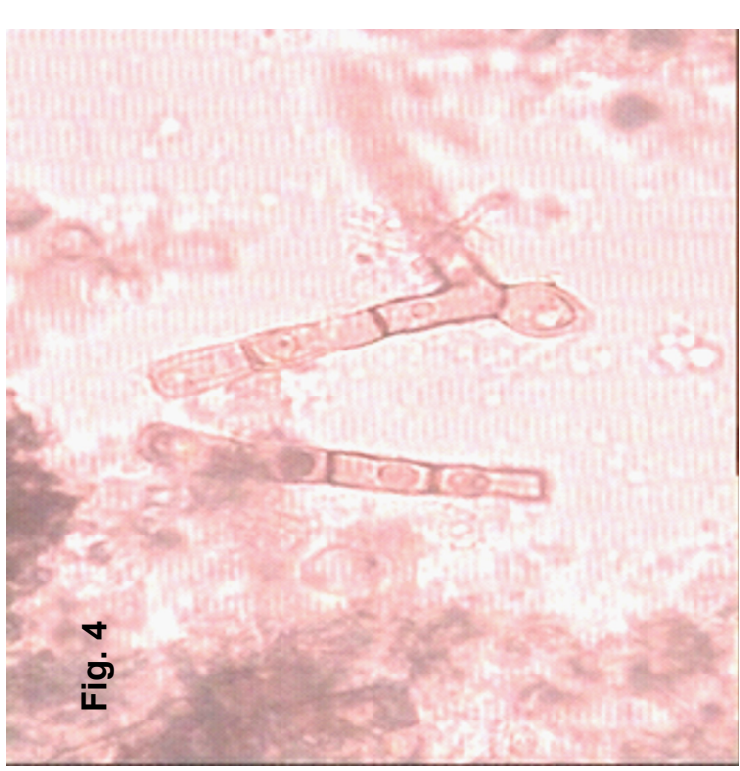

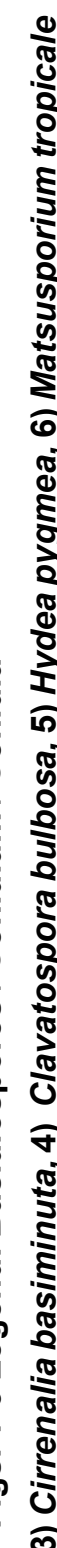

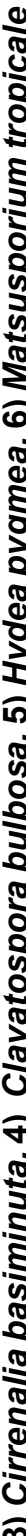




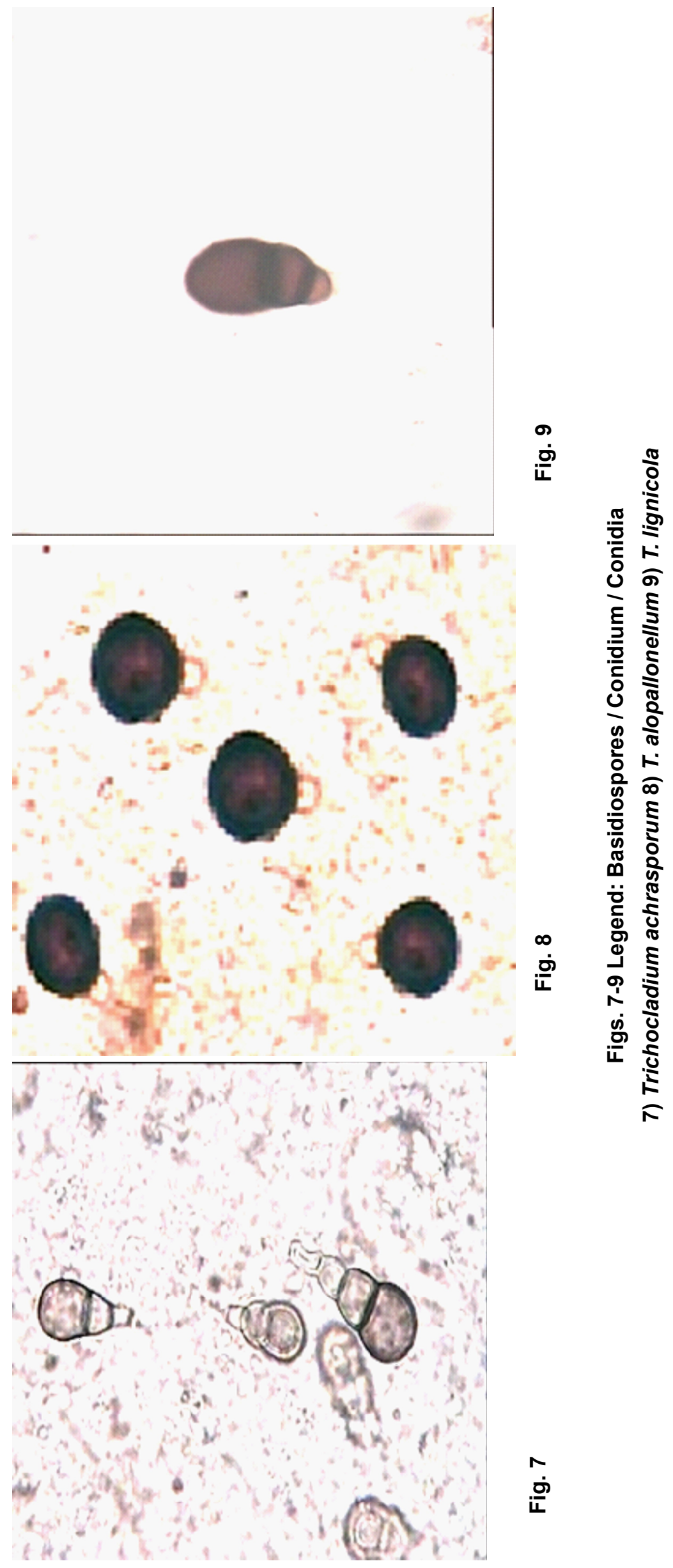


conidium.

Distribution in India:- West Coast: Gujarat, Maharashtra, Goa, Karnataka, Punducherry (Mahe), Kerala, Lakshadweep Islands; East Coast: Tamil Nadu, Andhra Pradesh, Odisha, West Bengal, Andaman \& Nicobar Islands (see, 2,3,\& 4).

9) Trichocladium lignicola I. Schmidt, 1985. Mycotaxon,
24: 419.

Conidia: $25-45 \times 8-20 \mu \mathrm{m}$, apical cell $15-20 \mu \mathrm{m}$ in diam, 2-4 septate, reddish-brown, markedly constricted at the septa, subglobose.

Distribution in India:- West Coast: Gujarat (see, $2,3, \& 4)$

\section{References}

1. Borse BD, Patil RV, Kelkar DJ. Marine fungi from Div Island (India). J. Adv. Sci. Tech. 1999; 11: 1-8.

2. Borse BD, Borse KN, Pawar NS, Tuwar AR. "Marine Fungi of India (Monograph)", Broadway Book Centre Publishers and Distributors, Panjim, Goa. 2012; pp. 1-471.

3. Borse BD, Borse KN, Pawar NS, Tuwar AR. Marine fungi from India -XII: A revised check list. Ind. J. Geo-Marine Sci., 2013; 42: 110-119.

4. Borse BD, Borse KN, Chaudhary SA, Patil VR, Patil SY, Gosavi SA, Borade DS. "Freshwater and Marine Fungi of India", LAP Lambert Academic Publishing, Germany. 2017; pp-1-163.

5. Borse BD, Pawar NS, Patil SY, Borse KN, Patil VR. Aquatic fungi of India: Freshwater and Marine. Bioinfolet. 2018; 15: 137-148.

6. Jamaluddhin S, Goswami MG, Ojha BM. "Fungi of India”, 1989-2001, Scientific Publishers, Jodhpur (India). p. 326.

7. Jones EBG. Fifty years of marine mycology. Fungal Diversity. 2011; 50: 73-112.

8. Jones EBG, Sakayaroj J, Suestrong S, Somrithipol S, Pang KL. Classification of Ascomycota, anamorphic taxa and Basidiomycota. Fungal Diversity. 2009; 35: 1-187.

9. Jones EBG, Suetrong S, Sakayaroj J, Bahkali AH, Abdel-Wahab MA, Boekhout T, Pang KL. Classification of marine Ascomycota, Basidiomycota, Blastocladiomycota and Chytridiomycota. Fungal Diversity. 2015; 73: $1-72$.

10. Volkmann-Kohlmeyer B, Kohlmeyer J. How to prepare truly permanent microscopic slides, Mycologist, 1996; 10: 107-108. 